\title{
MAXIMAL ABELIAN SUBALGEBRAS OF HYPERFINITE FACTORS
}

\author{
BY \\ OLE A. NIELSEN
}

1. Introduction. In this paper we shall be concerned primarily with constructing examples of maximal abelian von Neumann subalgebras (MA subalgebras) of hyperfinite factors of type III. Our results will show that certain phenomena known to hold for the hyperfinite factor of type $\mathrm{II}_{1}$ also hold for type III factors.

Let $\mathscr{M}$ and $\mathscr{N}$ be subalgebras of a factor $\mathscr{A}$. We call $\mathscr{M}$ and $\mathscr{N}$ equivalent (in $\mathscr{A})$ if $\mathscr{M}$ is the image of $\mathscr{N}$ by some automorphism of $\mathscr{A}$. Let $N(\mathscr{M})=N^{1}(\mathscr{M})$ be the subalgebra of $\mathscr{A}$ generated by all those unitary operators in $\mathscr{A}$ which induce automorphisms of $\mathscr{M}$, and for each integer $j \geqq 2$, let $N^{j}(\mathscr{H})=N\left(N^{j-1}(\mathscr{M})\right)$. Following Dixmier [4] and Anastasio [1], we call a MA subalgebra $\mathscr{H}$ of $\mathscr{L}$ singular if $N(\mathscr{M})=\mathscr{M}$, regular if $N(\mathscr{M})=\mathscr{A}$, semiregular if $N(\mathscr{H})$ is a factor distinct from $\mathscr{A}$, and $m$-semiregular $(m \geqq 2)$ if $N(\mathscr{H}), \ldots, N^{m-1}(\mathscr{M})$ are not factors but $N^{m}(\mathscr{H})$ is a factor. Generalizing the length of Tauer [11], we say that a MA subalgebra $\mathscr{H}$ of $\mathscr{A}$ has proper [improper] length $n$ if $n$ is the smallest positive integer such that $\dddot{I}^{n}(\mathscr{M})=N^{n+1}(\mathscr{M})$ and if $N^{n}(\mathscr{M})=\mathscr{A}\left[N^{n}(\mathscr{M}) \neq \mathscr{A}\right]$. As these properties are all equivalence-invariant, they can be used to establish the inequivalence of subalgebras.

In 1954 Dixmier [4] initiated the study of MA subalgebras of factors. He showed that the hyperfinite $\mathrm{II}_{1}$ factor $\mathscr{A}$ contains a regular, a semiregular, and a singular MA subalgebra. It is now known that $\mathscr{A}$ contains sequences of mutually inequivalent singular (Pukánszky [9]), semiregular (Tauer [11], [12]), and 2- and 3 -semiregular (Anastasio [1]) MA subalgebras; moreover, $\mathscr{A}$ contains an $n$-semiregular MA subalgebra for each $n \geqq 4$ (Tauer [13]). Both Tauer and Anastasio used the invariant of proper length to establish the mutual inequivalence of their sequences.

Maximal abelian subalgebras of type III factors have previously been considered by Pukánszky [8]. He obtained a general method for constructing MA subalgebras in a class of type III factors. However, there is an error in his calculation of the normalizer of these subalgebras, and so their types are not known.

Let $\mathscr{A}$ be one of the hyperfinite type III factors of Powers [7]. That $\mathscr{A}$ contains a regular MA subalgebra is trivial. Our main results are as follows:

THEOREM 1. $\mathscr{A}$ contains a semiregular MA subalgebra.

Received by the editors October 18, 1968 and, in revised form, February 11, 1969.

Copyright 01969 , American Mathematical Society 
THEOREM 2. For each choice of $n=2,3$ and $k=0,1,2, \ldots, \&$ contains an $n$ semiregular MA subalgebra of improper length $n+k$.

THEOREM 3. Theorem 2 remains true when "improper" is replaced by "proper".

THEOREM 4. For each choice of $n=2,3$ and $k=0,1,2, \ldots$, the hyperfinite $\mathrm{II}_{1}$ factor contains an n-semiregular MA subalgebra of improper length $n+k$.

The results in this paper are included in the author's doctoral thesis at the University of British Columbia. The author would like to thank Professor D. Bures, his advisor, for his assistance during the preparation of the thesis.

2. Notation and preliminaries. With the exception of group operator algebras and constructible algebras, our notation and terminology is taken from Dixmier's book [5].

Let $G$ be a group with identity $e$. We use $\bar{G}$ to denote the (complex) Hilbert space with orthonormal basis $(\bar{g})_{g \in G}$. For each $g \in G$ there is a unique unitary operator $V(g)$ on $\bar{G}$ satisfying

$$
V(g) \bar{h}=(g h)^{-} \text {for all } h \in G
$$

The group operator algebra over $G$, which we denote by $\mathscr{A}(G)$, is the von Neumann algebra on $\bar{G}$ generated by $\{V(g): g \in G\}$; it consists of all those operators $A$ on $\bar{G}$ with $(A \bar{g}, \bar{h})=\left(A \bar{e},\left(h g^{-1}\right)^{-}\right)$for all $g, h \in G$. A more complete discussion of the group operator algebra can be found in Murray and von Neumann [6, §5.3] or Dixmier [5, pp. 301-303]. For a subgroup $G_{0}$ of $G$, let $N\left(G_{0}\right)$ be the normalizer of $G_{0}$ in $G$ and let $\mathscr{M}\left(G, G_{0}\right)$ be the subalgebra of $\mathscr{A}(G)$ generated by $\left\{V(g): g \in G_{0}\right\}$; $\mathscr{A}\left(G, G_{0}\right)$ consists of all those operators $A$ in $\mathscr{A}(G)$ with $(A \bar{e}, \bar{g})=0$ for all $g \notin G_{0}$ (cf. [4, p. 280]).

LEMMA 2.1. If $G_{0}$ is a subgroup of the group $G$, then $\mathscr{A}\left(G_{0}\right) \cong \mathscr{M}\left(G, G_{0}\right)$.

Proof. We consider $\bar{G}_{0}$ to be a subspace of $\bar{G}$ in the obvious manner; then $\bar{G}_{0}$ is invariant under $\mathscr{M}\left(G, G_{0}\right)$ and the restriction map is the required isomorphism.

For our purposes the most important class of von Neumann algebras are the constructible algebras (for a full account, consult Dixmier [5, pp. 127-137]; see also Bures $[3, \S 1])$. We call $[\mathscr{M}, \mathscr{H}, G, g \rightarrow U(g)]$ a $C$-system if $\mathscr{M}$ is a maximal abelian von Neumann algebra on the Hilbert space $\mathscr{H}$, if $G$ is a group, and if $g \rightarrow U(g)$ is a unitary representation of $G$ on $\mathscr{H}$ such that $U(g) \mathscr{M} U(g)^{*}=\mathscr{M}$ for all $g \in G$. Let $[\mathscr{M}, \mathscr{H}, G, g \rightarrow U(g)]$ be a $C$-system. Finite linear combinations of the $M U(g) \otimes V(g), M \in \mathscr{M}$ and $g \in G$ (and $V(g)$ as in (2.1)), form a *algebra on $\mathscr{H} \otimes \bar{G}$; let $\mathscr{A}[\mathscr{M}, \mathscr{H}, G, g \rightarrow U(g)]$ denote the von Neumann algebra generated by this *-algebra. Bures calls $\mathscr{A}=\mathscr{A}[\mathscr{M}, \mathscr{H}, G, g \rightarrow U(g)]$ a constructible algebra if $\mathscr{M} \cap U(g) \mathscr{M}=\{0\}$ whenever $g \in G-\{e\}$ [3]. In what follows we shall frequently 
make use of the fact that $\mathscr{A}$ consists of all those operators $A$ on $\mathscr{H} \otimes \bar{G}$ such that for some (necessarily unique) $\mathscr{M}$-valued map $g \rightarrow A(g)$ on $G$,

$$
(A x \otimes \bar{g}, y \otimes \bar{h})=\left(A\left(h g^{-1}\right) U\left(h g^{-1}\right) x, y\right)
$$

for all $x, y \in \mathscr{H}$ and all $g, h \in G$. Dixmier [5, p. 129] has shown that every $A \in \mathscr{A}$ satisfies this condition. Conversely, suppose that an operator $A$ on $\mathscr{H} \otimes \bar{G}$ has this property. Let $W$ be the operator on $\mathscr{H} \otimes \bar{G}$ defined by $W x \otimes \bar{g}=U(g)^{*} x$ $\otimes\left(g^{-1}\right)^{-}, x \in \mathscr{H}$ and $g \in G$. Then by [5, p. 136, Exercise 1], it suffices to show that $A$ commutes with $W[M U(g) \otimes V(g)] W$ for all $M \in \mathscr{M}$ and $g \in G$. But this is an easy calculation. Moreover, for fixed $g \in G$, the map $A \rightarrow A(g)$ from $\mathscr{A}$ into $\mathscr{M}$ is linear and weakly continuous.

Lemma 2.2. Let $A, B \in \mathscr{A}$ ( $\mathscr{A}$ as in the previous paragraph), $g, h \in G$ and $M \in \mathscr{M}$ be given. Then:

(i) $[M U(h) \otimes V(h)](g)=\delta_{g, h} M$,

(ii) $A^{*}(g)=U(g)\left[A\left(g^{-1}\right)\right]^{*} U(g)$,

(iii) $(A B)(g)=\sum_{k \in G} A\left(g k^{-1}\right) U\left(g k^{-1}\right) B(k) U\left(g k^{-1}\right)^{*}$, the sum converging in the weak topology.

Proof. Observe first that for any $C \in \mathscr{A}$, the operator $C(g)$ is determined by the condition that

$$
(C x \otimes \bar{e}, y \otimes \bar{g})=(C(g) U(g) x, y) \text { for all } x, y \in \mathscr{H} .
$$

Let $x$ and $y$ be fixed but arbitrary elements of $\mathscr{H}$. Then

$$
(M U(h) \otimes V(h) x \otimes \bar{e}, y \otimes \bar{g})=((M U(h) x) \otimes \bar{h}, y \otimes \bar{g})=\left(\delta_{g, h} M U(g) x, y\right),
$$

which proves (i); (ii) follows by a similar computation. We now prove (iii). For each $k \in G$, let $E(k)$ denote the projection of $\mathscr{H} \otimes \bar{G}$ onto $\mathscr{H} \otimes[k]$; the $E(k)$ are mutually orthogonal and sum up to the identity. For all $k \in G, E(k) B x \otimes \bar{e}$ $=[B(k) U(k) x] \otimes k$ (take the inner product of both sides with $y \otimes k^{\prime}, k^{\prime} \in G$ arbitrary). Then

$$
\begin{aligned}
(A B x \otimes \bar{e}, y \otimes \bar{g}) & =\sum_{k \in G}(A E(k) B x \otimes \bar{e}, y \otimes \bar{g}) \\
& =\sum_{k \in G}(A[B(k) U(k) x \otimes \bar{k}], y \otimes \bar{g}) \\
& =\sum_{k \in G}\left(A\left(g k^{-1}\right) U\left(g k^{-1}\right) B(k) U\left(g k^{-1}\right)^{*} U(g) x, y\right) .
\end{aligned}
$$

Lemma 2.3. Let $[\mathcal{H}, \mathscr{H}, G, g \rightarrow U(g)]$ be a $C$-system and let

$$
\mathscr{A}=\mathscr{A}[\mathscr{M}, \mathscr{H}, G, g \rightarrow U(g)] \text {. }
$$

Let $\mathscr{M}_{0}$ be a subalgebra of $\mathscr{M}$, let $G_{0}$ be a subgroup of $G$, and suppose that

$$
U(g) \mathscr{H}_{0} U(g)^{*}=\mathscr{H}_{0}
$$


for all $g \in G_{0}$. Then the von Neumann algebra generated by the $M U(g) \otimes V(g)$, $M \in \mathscr{M}_{0}$ and $g \in G_{0}$, consists of all those operators $A \in \mathscr{A}$ such that

(i) $A(g) \in \mathscr{M}_{0}$ whenever $g \in G_{0}$,

(ii) $A(g)=0$ whenever $g \in G-G_{0}$.

Proof. Let $\mathscr{A}_{0}=\mathscr{R}\left(M U(g) \otimes V(g): M \in \mathscr{M}_{0}, g \in G_{0}\right)$ and let $\mathscr{A}_{1}=\{A \in \mathscr{A}: A$ satisfies (i) and (ii) $\}$. From the weak continuity of $A \rightarrow A(g)$ and the hypothesis, it follows that $\mathscr{A}_{1}$ is a von Neumann algebra containing $\mathscr{A}_{0}$. To show that $\mathscr{A}_{1} \subset \mathscr{A}_{0}$, let a $T \in \mathscr{A}_{0}^{\prime}$ and an $A \in \mathscr{A}_{1}$ be given. Then, for all $x, y \in \mathscr{H}$ and $g, h \in G$,

$$
\begin{aligned}
(T A x \otimes \bar{g}, y \otimes \bar{h}) & =\sum_{k \in G}\left(E(k g) A x \otimes \bar{g}, T^{*} y \otimes \bar{h}\right) \\
& =\sum_{k \in G}\left(A(k) U(k) x \otimes(k g)^{-}, T^{*} y \otimes \bar{h}\right) \\
& =\sum_{k \in G}(T[A(k) U(k) \otimes V(k)] x \otimes \bar{g}, y \otimes \bar{h}) \\
& =\sum_{k \in G}\left(T x \otimes \bar{g}, A^{*}(k) U(k) y \otimes(k h)^{-}\right) \\
& =\sum_{k \in G}\left(T x \otimes \bar{g}, E(k h) A^{*} y \otimes \bar{h}\right) \\
& =(A T x \otimes \bar{g}, y \otimes \bar{h}) .
\end{aligned}
$$

Therefore $\mathscr{A}_{0}^{\prime} \subset \mathscr{A}_{1}^{\prime}$, and so $\mathscr{A}_{0}=\mathscr{A}_{1}$.

The next result, which is of a technical nature, will be used only in the proof of Lemma 3.11.

LEMMA 2.4. Let $\mathscr{M}$ be an abelian von Neumann algebra acting on the Hilbert space $\mathscr{H}$ and let $x$ be a unit vector in $\mathscr{H}$. Let $\left(M_{i}\right)_{i \in I}$ and $\left(N_{i}\right)_{i \in I}$ be two families in $\mathscr{M}$ such that $M=\sum_{i \in I} M_{i} N_{i}$ and $N=\sum_{i \in I} N_{i} N_{i}^{*}$ exist in $\mathscr{M}$ in the strong topology, and suppose that $N \leqq I$. Then $\|M x\|^{2} \leqq \sum_{i \in I}\left\|M_{i} x\right\|^{2}$.

Proof. By the Gelfand-Naimark representation theorem, there is a compact Hausdorff space $X$ such that $\mathscr{M}$ and $C(X)$ are isomorphic as $C^{*}$-algebras. Let $f, g, f_{i}, g_{i}$ in $C(X)$ correspond to $M, N, M_{i}, N_{i}(i \in I)$ in $\mathscr{M}$, resp. Fix a finite subset $F$ of $I$. As $0 \leqq \sum_{i \in F} N_{i} N_{i}^{*} \leqq N \leqq I, 0 \leqq \sum_{i \in F}\left|g_{i}\right|^{2} \leqq 1$. Then

$$
\left|\sum_{i \in F} f_{i} g_{i}\right|^{2} \leqq\left(\sum_{i \in F}\left|f_{i}\right|^{2}\right)\left(\sum_{i \in F}\left|g_{i}\right|^{2}\right) \leqq \sum\left|f_{i}\right|^{2}
$$

by the usual Cauchy-Schwarz inequality. Therefore

$$
\left(\sum_{i \in F} M_{i} N_{i}\right)^{*}\left(\sum_{i \in F} M_{i} N_{i}\right) \leqq \sum_{i \in F} M_{i}^{*} M_{i}
$$

which in turn implies that $\left\|\sum_{i \in F} M_{i} N_{i} x\right\|^{2} \leqq \sum_{i \in F}\left\|M_{i} x\right\|^{2}$. As $F$ is a finite but otherwise arbitrary subset of $I$, and as $M=\sum_{i \in F} M_{i} N_{i}$ strongly, we are done. 
3. The main construction. Throughout this section $p$ will denote a fixed point in $\left(0, \frac{1}{2}\right)$ and $G$ will denote a fixed countably infinite group with identity $e$. In order to simplify the notation, dependence on $p$ and $G$ will often not be indicated.

Let $\Delta$ denote the abelian group (under pointwise addition modulo 2 ) of all those $\alpha: G \rightarrow\{0,1\}$ such that $\alpha(g)=0$ for all but finitely many $g \in G$. For $g \in G$ and $\alpha \in \Delta$ let $g \alpha$ and $\alpha g$ be those elements of $\Delta$ defined by

$$
\begin{aligned}
(g \alpha)(h) & =\alpha\left(g^{-1} h\right) & & h \in G, \\
(\alpha g)(h) & =\alpha(h) & & h \neq g, \\
& =1-\alpha(g) & & h=g,
\end{aligned}
$$

and let $\leqq$ denote the pointwise partial ordering in $\Delta$. Let $\mathscr{H}$ denote the Hilbert space with orthonormal basis $\left(\phi_{\alpha}\right)_{\alpha \in \Delta}$. For each $g \in G$ define operators $U(g), F(g)$ and $W(g)$ on $\mathscr{H}$ by the relations

$$
\begin{aligned}
U(g) \phi_{\alpha} & =\phi_{g \alpha}, \\
F(g) \phi_{\alpha} & =[(1-2 p) \alpha(g)+p] \phi_{\alpha}+[p(1-p)]^{1 / 2} \phi_{\alpha g}, \\
W(g) \phi_{\alpha} & =2(-1)^{\alpha(g)}[p(1-p)]^{1 / 2} \phi_{\alpha}+(1-2 p) \phi_{\alpha g} .
\end{aligned}
$$

Lemma 3.1. $g \rightarrow U(g)$ is a unitary representation of $G$ on $\mathscr{H},\{F(g): g \in G\}$ and $\{W(g): g \in G\}$ are commuting families of projections and selfadjoint unitaries, resp., and for all $g, h \in G$,

$$
\begin{aligned}
U(g) F(h) U(g)^{*} & =F(g h), & & \\
W(g) F(h) W(g) & =F(h) & & h \neq g, \\
& =I-F(h) & h & =g .
\end{aligned}
$$

Proof. Compute.

Let $P_{0}=W(0)=I$, and for each $g \in G$ and $\alpha \in \Delta-\{0\}$, let

$$
\begin{aligned}
P_{g} & =((1-p) / p)^{1 / 2} F(g)-(p /(1-p))^{1 / 2}(I-F(g)), \\
P_{\alpha} & =\prod_{\alpha(g)=1} P_{g}, \\
W(\alpha) & =\prod_{\alpha(g)=1} W(g)
\end{aligned}
$$

(both products are finite with commuting factors). As $P_{\alpha} \phi_{0}=\phi_{\alpha}$ for each $\alpha \in \Delta$, $\mathscr{M}=\mathscr{R}(F(g): g \in G)$ is a MA von Neumann algebra on $\mathscr{H}$ [5, p. 109, Exercise 5]. From Lemma $3.1, \alpha \rightarrow W(\alpha)$ is a unitary representation of $\Delta$ on $\mathscr{H}$ such that $W(\alpha) \mathscr{M} W(\alpha)=\mathscr{M}$ for each $\alpha \in \Delta$. Let $\mathscr{A}_{p}=\mathscr{A}[\mathscr{M}, \mathscr{H}, \Delta, \alpha \rightarrow W(\alpha)]$ (notice that $\mathscr{A}_{p}$ does not depend on the choice of the group $G$ ). There are several ways of seeing that $\mathscr{A}_{p}, 0<p<\frac{1}{2}$, is the one-parameter family of mutually nonisomorphic hyperfinite type III factors of Powers [7]. Perhaps the simplest way is the following (cf. $[7, \S 4]):$ Consider the $C^{*}$-algebra $\mathfrak{A}_{p}$ on $\mathscr{H} \otimes \bar{\Delta}$ generated by

$$
\{M W(\alpha) \otimes V(\alpha): M \in \mathscr{M}, \alpha \in \Delta\}
$$


and for each $g \in G$, the subalgebra $\mathscr{M}_{g}$ generated by $F(g) W(g) \otimes V(0 g)$. Each $\mathscr{M}_{g}$ is a factor of type $\mathrm{I}_{2}$ and $\left\{\mathscr{M}_{\mathrm{g}}: g \in G\right\}$ is a factorization of $\mathfrak{N}_{p}$. The state on $\mathfrak{A}_{p}$ induced by $\phi_{0} \otimes \overline{0}$ has all the properties of the state $\omega_{p}$ of [7, Definition 4.2]. As $\phi_{0} \otimes \overline{0}$ is cyclic for $\mathfrak{A}_{p}$, the representation of $\mathfrak{A}_{p}$ induced by this state is spatially isomorphic to $\mathfrak{A}_{p}[5$, p. 51]. A second proof (cf. [2, Definition 3.10]) consists in verifying that

$$
\mathscr{A}_{p} \cong \bigotimes_{n=1}^{\infty}\left(\mathscr{L}\left(\mathscr{H}_{n}\right) \otimes C_{\mathscr{X}_{n}},(1-p)^{1 / 2} x_{n} \otimes s_{n}+p^{1 / 2} y_{n} \otimes t_{n}\right)
$$

where, for each $n, \mathscr{H}_{n}$ and $\mathscr{K}_{n}$ are 2-dimensional Hilbert spaces with orthonormal basis $\left\{x_{n}, y_{n}\right\}$ and $\left\{s_{n}, t_{n}\right\}$, resp.

Lemma 3.2. $\mathscr{N}=\mathscr{M} \otimes C_{\bar{\Delta}}$ is a regular $M A$ subalgebra of $\mathscr{A}_{p}$.

Proof. To show that $\mathscr{N}$ is MA in $\mathscr{A}_{p}$ it suffices, by $[5$, p. 131], to show that $W(\alpha) \mathscr{M} \cap \mathscr{M}=\{0\}$ for all nonzero $\alpha \in \Delta$. If this is not the case, then $W(\alpha) M \in \mathscr{M}$ for some nonzero $M \in \mathscr{M}$ and some nonzero $\alpha \in \Delta$. Choosing a $g \in G$ such that $\alpha(g)=1$, we obtain

$$
\begin{aligned}
0 & =\left\|(F(g) W(\alpha) M-W(\alpha) M F(g)) \phi_{0}\right\| \\
& =\left\|(I-2 F(g)) M \phi_{0}\right\| \\
& =\left\|M \phi_{0}\right\|,
\end{aligned}
$$

which contradicts the fact that $\phi_{0}$ is separating for $\mathscr{M}$. Finally, it is clear that $\mathscr{M}$ is regular in $\mathscr{A}_{p}$.

LEMMA 3.3. The set of all finite linear combinations of the $P_{\alpha}, \alpha \in \Delta$, is a strongly dense sub*-algebra of $\mathscr{M}$.

Proof. It is clear that the set $\mathscr{S}$ of all finite linear combinations of the $P_{\alpha}(\alpha \in \Delta)$ is a linear space, that $\mathscr{S}=\mathscr{S}^{*}$, and that $\mathscr{S}$ contains the identity as well as each $F(g)$. A simple calculation shows that $P_{a}^{2} \in \mathscr{S}$ for all $\alpha \in \Delta$. As $P_{\alpha} P_{\beta}=P_{\alpha+\beta} P_{\alpha \beta}^{2}, \alpha, \beta \in \Delta$, (where $\alpha \beta$ denotes the pointwise product of $\alpha$ and $\beta$ ) and as $(\alpha+\beta)(g)=1$ implies $(\alpha \beta)(g)=0, \mathscr{S}$ is closed under multiplication. An application of the double commutant theorem now completes the proof.

LEMMA 3.4. If an $M \in \mathscr{M}$ commutes with $U(g)$ for infinitely many $g \in G$, then $M \in C_{\mathscr{H}}$.

Proof. Suppose that $M \in \mathscr{M}$ satisfies the hypothesis. If $M \phi_{0}=\sum_{\alpha \in \Delta} c_{\alpha} \phi_{\alpha}$, then $c_{\sigma \alpha}=c_{\alpha}$ for all $\alpha \in \Delta$ and infinitely many $g \in G$. But this implies that $M \phi_{0}=c_{0} \phi_{0}$, and thus $M=c_{0} I$.

Lemma 3.5. For each $g \in G-\{e\}, \mathscr{M} \cap U(g) \mathscr{M}=\{0\}$.

Proof. Suppose that $U(g) M \in \mathscr{M}$ for some $g \in G-\{e\}$ and some nonzero $M \in \mathscr{M}$. Choose an $\varepsilon$ with $0<2 \varepsilon^{2}<p-p^{2}$ and a nonzero $S \in \mathscr{S}$ with

$$
\left\|(M-S) \phi_{0}\right\|<\varepsilon\left\|M \phi_{0}\right\| /(1+\varepsilon)
$$


( $\mathscr{S}$ as in Lemma 3.3). Write $S S^{*}=\sum_{\alpha \in \Gamma} c_{\alpha} P_{\alpha}$ for some finite $\Gamma \subset \Delta$ and complex numbers $c_{\alpha}$. Choosing an $h \in G-\{e\}$ such that $\alpha(h)=\alpha\left(g^{-1} h\right)=0$ for all $\alpha \in \Gamma$,

$$
\begin{aligned}
\left\|[F(h) U(g) S-U(g) S F(h)] \phi_{0}\right\|^{2} & =\left\|\left[F\left(g^{-1} h\right) S-S F(h)\right] \phi_{0}\right\|^{2} \\
& =2\left(p-p^{2}\right)\left\|S \phi_{0}\right\|^{2}
\end{aligned}
$$

on the other hand,

$$
\begin{aligned}
\left\|[F(h) U(g) S-U(g) S F(h)] \phi_{0}\right\| \leqq & \left\|[F(h) U(g) S-F(h) U(g) M] \phi_{0}\right\| \\
& +\left\|[U(g) M F(h)-U(g) S F(h)] \phi_{0}\right\| \\
\leqq & 2\left\|(M-S) \phi_{0}\right\| \\
\leqq & 2 \varepsilon\left(\left\|M \phi_{0}\right\|-\varepsilon\left\|M \phi_{0}\right\| /(1+\varepsilon)\right) \\
\leqq & 2 \varepsilon\left\|S \phi_{0}\right\| .
\end{aligned}
$$

Therefore $2 \varepsilon^{2} \geqq p-p^{2}$, a contradiction.

As $U(g) \mathscr{M} U(g)^{*}=\mathscr{M}$ for each $g \in G$ (Lemma 3.1), we can make the

Definition 3.6. Let $\mathscr{B}(G)=\mathscr{A}[\mathscr{H}, \mathscr{H}, G, g \rightarrow U(g)]$, and for each subgroup $G_{0}$ of $G$, let $\mathscr{N}\left(G, G_{0}\right)=\mathscr{R}\left(U(g) \otimes V(g): g \in G_{0}\right)$.

Proposition 3.7. $\mathscr{B}(G)$ is a factor of type $\mathrm{II}_{1}$ and is hyperfinite whenever $G$ is the union of an increasing sequence of finite subgroups.

Proof. The trace on $\mathscr{M}^{+}$induced by $\phi_{0} \otimes \bar{e}$, Lemmas 3.4 and 3.5 and [5, pp. 127-132] imply that $\mathscr{B}(G)$ is a finite factor. From [5, p. 136, Exercise 2], and the fact that $\mathscr{A}_{p}$ is of type III, there can be no minimal projections in $\mathscr{B}(G)$. Therefore $\mathscr{B}(G)$ is not of type $\mathrm{I}$, and must therefore be of type $\mathrm{II}_{1}$.

If $G=\bigcup_{n=1}^{\infty} G_{n}$, where $G_{1}, G_{2}, \ldots$ is an increasing sequence of finite subgroups of $G$, then the $\mathscr{B}_{n}=\mathscr{R}\left(F(h) U(g) \otimes V(g): g, h \in G_{n}\right)$ form an increasing sequence of finite-dimensional *-algebras which generate $\mathscr{B}(G)$, and therefore $\mathscr{B}(G)$ is hyperfinite [5], [6].

From now on we will frequently be replacing an operator $A$ in a constructible algebra by its associated operator-valued function $g \rightarrow A(g)$ (cf. $§ 2)$.

LEMMA 3.8. Let $G_{0}$ be an abelian subgroup of $G$ such that $\left\{g^{-1}: g \in G_{0}\right\}$ is infinite whenever $h \in G-G_{0}$. Then $\mathscr{N}\left(G, G_{0}\right)$ is $M A$ in $\mathscr{B}(G)$.

Proof. Clearly $\mathscr{N}\left(G, G_{0}\right)$ is abelian; to show that it is MA in $\mathscr{B}(G)$, let a $B \in \mathscr{B}(G) \cap\left(\mathscr{N}\left(G, G_{0}\right)\right)^{\prime}$ be given. By Lemma 2.3 , it suffices to show that $B(h) \in C_{\mathscr{H}}$ for all $h \in G_{0}$ and that $B(h)=0$ for all $h \in G-G_{0}$. First of all,

$$
\left(\left(B B^{*}\right)(e) \phi_{0}, \phi_{0}\right)=\sum_{g \in G}\left\|B(g) \phi_{0}\right\|^{2}<\infty ;
$$

moreover, for all $g \in G_{0}$ and $h \in G$, the expressions

$$
\begin{aligned}
& (B[U(g) \otimes V(g)])(h g)=B(h), \\
& ([U(g) \otimes V(g)] B)(h g)=U(g) B\left(g^{-1} h g\right) U(g)^{*}
\end{aligned}
$$


are equal. Now if $h \in G_{0}, B(h)=U(g) B(h) U(g) *$ for all $g \in G_{0}$, and so $B(h) \in C_{\mathscr{H}}$ by Lemma 3.4. And if $h \in G-G_{0},\left\|B(h) \phi_{0}\right\|=\left\|B\left(g^{-1} h g\right) \phi_{0}\right\|$ for all $g \in G_{0}$; by the hypothesis of the Lemma and (3.1), $B(h) \phi_{0}=0$, and consequently $B(h)=0$.

REMARK. The converse of Lemma 3.8 is also true, but as we will not need it, we omit the proof.

Lemma 3.9. There is a *-isomorphism $\Phi$ of $\mathscr{A}(G)$ onto $\mathscr{N}(G, G)$ such that $\Phi(V(g))=U(g) \otimes V(g)$ for all $g \in G$.

Proof. Let $\eta$ be the unitary operator on $\mathscr{H} \otimes \bar{G}$ defined by the relations $\eta\left(\phi_{\alpha} \otimes \bar{g}\right)=\phi_{g \alpha} \otimes \bar{g}, \alpha \in \Delta$ and $g \in G$, and for each $T \in \mathscr{A}(G)$, let $\Phi(T)=\eta(I \otimes T) \eta^{-1}$; then $\Phi$ is a normal *-isomorphism [5, p. 56, Definition 2] of $\mathscr{A}(G)$ into $\mathscr{L}(\mathscr{H} \otimes \bar{G})$. That $\Phi(V(g))=U(g) \otimes V(g), g \in G$, is a simple computation. By [5, p. 57], we would be done if we could show that $\Phi(\mathscr{A}(G)) \subset \mathscr{N}(G, G)$. But this follows from the easily-verified identity

$$
\left(\Phi(T) \phi_{\alpha} \otimes \bar{g}, \phi_{\beta} \otimes \bar{h}\right)=\left(\left(T \bar{e},\left(h g^{-1}\right)^{-}\right) U\left(h g^{-1}\right) \phi_{\alpha}, \phi_{\beta}\right),
$$

valid for all $\alpha, \beta \in \Delta$, all $g, h \in G$, and all $T \in \mathscr{A}(G)$.

Let $\mathscr{G}=\Delta \times G$ (the group-theoretic direct product), and for $a=(\alpha, g) \in \mathscr{G}$, let $U(a)=W(\alpha) \otimes U(g)$. In order to simplify the notation, we will write $T(a)$ instead of $U(a) \otimes V(a), a \in \mathscr{G}$. Since $\phi_{0} \otimes \phi_{0}$ is cyclic for $\mathscr{M} \otimes \mathscr{M}, \mathscr{M} \otimes \mathscr{M}$ is MA on $\mathscr{H} \otimes \mathscr{H}$; moreover, $a \rightarrow U(a)$ is a unitary representation of $\mathscr{G}$ on $\mathscr{H} \otimes \mathscr{H}$ such that $U(a) \mathscr{M} \otimes \mathscr{M} U(a)^{*}=\mathscr{M} \otimes \mathscr{M}$ for all $a \in \mathscr{G}$. Define $\mathscr{A}$ to be the von Neumann algebra $\mathscr{A}[\mathscr{M} \otimes \mathscr{M}, \mathscr{H} \otimes \mathscr{H}, \mathscr{G}, a \rightarrow U(a)]$ on $\mathscr{H} \otimes \mathscr{H} \otimes \mathscr{G}$. Let $\psi$ be the unique linear isometry of $\mathscr{H} \otimes \bar{\Delta} \otimes \mathscr{H} \otimes \bar{G}$ onto $\mathscr{H} \otimes \mathscr{H} \otimes \overline{\mathscr{G}}$ satisfying

$$
\psi\left(\phi_{\alpha} \otimes \bar{\gamma} \otimes \phi_{\beta} \otimes \bar{g}\right)=\phi_{\alpha} \otimes \phi_{\beta} \otimes(\gamma, g)^{-} \quad \text { for all } \alpha, \beta, \gamma \in \Delta \text { and } g \in G .
$$

Then

$$
\psi([M W(\alpha) \otimes V(\alpha)] \otimes[N U(g) \otimes V(g)]) \psi^{-1}=(M \otimes N) U(a) \otimes V(a)
$$

for all $M, N \in \mathscr{M}$ and $a=(\alpha, g) \in \mathscr{G}$, and consequently $A \rightarrow \psi A \psi^{-1}$ is a *-isomorphism of $\mathscr{A}_{p} \otimes \mathscr{B}(G)$ onto $\mathscr{A}$ (use [5, p. 57]).

Definition 3.10. For each subgroup $G_{0}$ of $G$, set $\mathscr{P}\left(G_{0}\right)=\psi \mathscr{N} \otimes \mathscr{N}\left(G, G_{0}\right) \psi^{-1}$ $\left(\mathscr{N}\right.$ as in Lemma 3.2) and set $\mathscr{S}\left(G_{0}\right)=\psi \mathscr{A}_{p} \otimes \mathscr{N}\left(G, G_{0}\right) \psi^{-1}$.

REMARK. As $\mathscr{N}(G, G)$ is a proper subalgebra of $\mathscr{B}(G), \mathscr{S}(G)$ is a proper subalgebra of $\mathscr{A}$.

Lemma 3.11 (CF. [8, Lemma 15]). Let a unitary $A \in \mathscr{A}$ and an $\varepsilon>0$ be given. Then there is a finite subset $\mathscr{F}_{0}$ of $\mathscr{G}$ such that if $\mathscr{F}$ is a finite subset of $\mathscr{G}$ containing $\mathscr{F}_{0}$, then there is a family $(R(b))_{b \in \mathscr{F}}$ of finite linear combinations of the $P_{\alpha} \otimes P_{\beta}$ $(\alpha, \beta \in \Delta)$ such that if $R=\sum_{b \in \mathscr{F}} R(b) U(b) \otimes V(b)$, then

$$
\left\|(A(b)-R(b)) \phi_{0} \otimes \phi_{0}\right\| \leqq \varepsilon / 2 \text { for all } b \in \mathscr{F},
$$


and moreover, for all $c, d \in\{0\} \times G$,

$$
\left\|\left(A T(d) A^{*}-R T(d) R^{*}\right)(c) \phi_{0} \otimes \phi_{0}\right\| \leqq \varepsilon^{2} / 4
$$

Proof. As

$$
I=\left(A A^{*}\right)((0, e))=\sum_{a \in \mathscr{G}} A(a) A(a)^{*}
$$

in the weak topology on $\mathscr{A}$ (cf. (3.1)), there is a finite subset $\mathscr{F}_{0}$ of $\mathscr{G}$ such that

$$
\sum_{a \notin \mathcal{F}}\left\|A(a) \phi_{0} \otimes \phi_{0}\right\|^{2} \leqq \varepsilon^{4} / 256
$$

Fix elements $c, d \in\{0\} \times G$ and a finite subset $\mathscr{F}$ of $\mathscr{G}$ containing $\mathscr{F}_{0}$. A calculation using Lemma 2.2 shows that

$$
\left(A T(d) A^{*}\right)(c)=\sum_{a \in \mathcal{G}} A(a) U(c) A\left(c^{-1} a d\right)^{*} U(c)^{*}
$$

in the weak topology; similarly, if $B=\sum_{b \in \mathscr{F}} A(b) U(b) \otimes V(b)$,

$$
\left(B T(d) B^{*}\right)(c)=\sum_{a \in \overline{\mathscr{J}}} A(a) U(c) A\left(c^{-1} a d\right)^{*} U(c)^{*},
$$

where $\mathscr{F}^{\prime}=\mathscr{F} \cap c \mathscr{F} d^{-1}$ (the empty sum is defined to be zero). We now find that

$$
\begin{aligned}
\left\|\left(A T(d) A^{*}-B T(d) B^{*}\right)(c) \phi_{0} \otimes \phi_{0}\right\| \leqq & \left\|\sum_{a \in \mathscr{F}^{*}-\mathcal{F}^{\prime}} A(a) U(c) A\left(c^{-1} a d\right)^{*} U(c)^{*} \phi_{0} \oplus \phi_{0}\right\| \\
& +\left\|\sum_{a \notin \mathcal{F}^{\mathcal{F}}} A(a) U(c) A\left(c^{-1} a d\right)^{*} U(c)^{*} \phi_{0} \otimes \phi_{0}\right\| \\
\leqq & \left(\sum_{a \in \mathscr{F}^{\prime}-\mathscr{F}^{\prime}}\left\|U(c) A\left(c^{-1} a d\right)^{*} U(c)^{*} \phi_{0} \otimes \phi_{0}\right\|^{2}\right)^{1 / 2} \\
& +\left(\sum_{a \notin \mathscr{F}}\left\|A(a) \phi_{0} \otimes \phi_{0}\right\|^{2}\right)^{1 / 2} \\
\leqq & 2\left(\sum_{a \notin \mathcal{F}^{\mathscr{F}}}\left\|A(a) \phi_{0} \otimes \phi_{0}\right\|^{2}\right)^{1 / 2} \\
\leqq & \varepsilon^{2} / 8
\end{aligned}
$$

where Lemma 2.4 has been applied twice.

By (3.4) each $A(a)$ is in the unit ball of $\mathscr{H} \otimes \mathscr{M}$. Hence (by Lemma 3.3 and the Kaplansky density theorem) there is a family $(R(b))_{b \in \mathcal{F}}$ of finite linear combinations of the $P_{\alpha} \otimes P_{\beta}(\alpha, \beta \in \Delta)$, each of which is in the unit ball of $\mathscr{M} \otimes \mathscr{M}$ and satisfies

$$
\left\|(A(b)-R(b)) \phi_{0} \otimes \phi_{0}\right\| \leqq \min \left(\varepsilon / 2, \varepsilon^{2} / 16 n\right) \text { for all } b \in \mathscr{F},
$$


where $n$ is the cardinality of $\mathscr{F}$. An easy computation now shows that (cf. (3.5))

$$
\begin{aligned}
&\left\|\left(B T(d) B^{*}-R T(d) R^{*}\right)(c) \phi_{0} \otimes \phi_{0}\right\| \\
& \leqq \sum_{a \notin \mathscr{F}^{\prime}} A(a) U(c)\left[A\left(c^{-1} a d\right)-R\left(c^{-1} a d\right)\right]^{*} U(c)^{*} \phi_{0} \otimes \phi_{0} \\
& \quad+\sum_{a \in \mathscr{F}^{\prime}}[A(a)-R(a)] U(c) R\left(c^{-1} a d\right)^{*} U(c)^{*} \phi_{0} \otimes \phi_{0} \| \\
& \leqq \varepsilon^{2} / 8 .
\end{aligned}
$$

An obvious application of the triangle inequality now completes the proof.

Lemma 3.12 (CF. [8, Lemma 17]). Suppose that $G_{0}$ is a subgroup of $G$ such that: given a finite subset $F$ of $G$ and a $g \in G$, there are infinitely many $g_{0} \in G_{0}$ such that

(i) $h, k \in F$ and $h g_{0} k^{-1}=g_{0}$ imply $h=k$,

(ii) if $g \notin N\left(G_{0}\right)$, then $g g_{0} g^{-1} \notin G_{0}$.

Then $N\left(\mathscr{P}\left(G_{0}\right)\right)=N\left(\mathscr{S}\left(G_{0}\right)\right)=\mathscr{S}\left(N\left(G_{0}\right)\right)$.

Proof. That $\mathscr{S}\left(N\left(G_{0}\right)\right) \subset N\left(\mathscr{P}\left(G_{0}\right)\right)$ and $\mathscr{S}\left(N\left(G_{0}\right)\right) \subset N\left(\mathscr{S}\left(G_{0}\right)\right)$ are clear. To show that the opposite inclusions also hold, suppose that we are given a unitary operator $A \in \mathscr{A}$ satisfying either

$$
A \mathscr{P}\left(G_{0}\right) A^{*}=\mathscr{P}\left(G_{0}\right) \text { or } A \mathscr{T}\left(G_{0}\right) A^{*}=\mathscr{S}\left(G_{0}\right)
$$

For each $a \in \mathscr{G}$ write

$$
A(a) \phi_{0} \otimes \phi_{0}=\sum_{\alpha, \beta \in \Delta} \theta(a ; \alpha, \beta) \phi_{\alpha} \otimes \phi_{\beta},
$$

where the $\theta(a ; \alpha, \beta)$ are complex. As the conditions

$$
\begin{aligned}
& \theta(a ; \alpha, \beta)=0 \quad \text { whenever } \beta \neq 0, \\
& \theta(a ; \alpha, 0)=0 \quad \text { whenever } a \notin \Delta \times N\left(G_{0}\right)
\end{aligned}
$$

together with Lemma 2.3 imply that $A \in \mathscr{S}\left(N\left(G_{0}\right)\right)$, it is sufficient to establish (3.7) and (3.8).

Fix an $(\alpha, \beta) \in \Delta \times \Delta$, an $a=(\gamma, g) \in \mathscr{G}$, and an $\varepsilon>0$. Lemma 3.11 applied to $A$ and $\varepsilon$ yields a finite subset $\mathscr{F}$ of $\mathscr{G}$ containing $a$ and operators

$$
R(b)=\sum_{\delta, \eta \in \Gamma} \sigma(b ; \delta, \eta) P_{\delta} \otimes P_{\eta} \quad(b \in \mathscr{F})
$$

satisfying (3.2) and (3.3), where $\Gamma$ is a finite subset of $\Delta$ containing $\alpha$ and $\beta$ and the $\sigma(b ; \delta, \eta)$ are complex numbers. In particular,

$$
|\theta(b ; \delta, \eta)-\sigma(b ; \delta, \eta)| \leqq\left\|(A(b)-R(b)) \phi_{0} \otimes \phi_{0}\right\| \leqq \varepsilon / 2
$$

for all $b \in \mathscr{F}$ and $\delta, \eta \in \Gamma$. Applying the hypothesis on $G_{0}$ to

$$
F=\left\{g^{-1} h:(\delta, h) \in \mathscr{F} \text { for some } \delta \in \Gamma\right\}
$$


and $g$, we obtain a $g_{0} \in G_{0}$ such that (i) and (ii) hold and in addition $k \in G, \delta \in \Gamma$ and $\delta(k)=1$ imply $\left(g g_{0} g^{-1} \eta\right)(k)=0$ for all $\eta \in \Gamma$.

In order to simplify the notation, set $h=g g_{0} g^{-1}, c=(0, h), d=\left(0, g_{0}\right)$, and set $\delta=\beta+h \beta$. Let $R \in \mathscr{A}$ be as in Lemma 3.11, let $\mathscr{F}{ }^{\prime}=\mathscr{F} \cap c \mathscr{F} d^{-1}$ (notice that $a \in \mathscr{F}^{\prime}$ ), and let $S=\left(R T(d) R^{*}\right)(c)$. By our choice of $g_{0}, b \in \mathscr{F}^{\prime}$ implies $c^{-1} b d=b$, and therefore (cf. (3.5))

$$
S=\sum_{b \in \mathcal{F}^{\prime}} R(b) U(c) R(b)^{*} U(c)^{*} .
$$

Again by our choice of $g_{0}$, if $\alpha_{1}, \alpha_{2}, \alpha_{3}, \alpha_{4} \in \Gamma$, then

$$
\begin{aligned}
& \left(P_{\alpha_{1}} P_{\alpha_{2}} \otimes P_{\alpha_{3}} U(h) P_{\alpha_{4}} U(h)^{*} \phi_{0} \otimes \phi_{0}, \phi_{0} \otimes \phi_{\delta}\right)=\left(P_{\alpha_{1}} P_{\alpha_{2}} \phi_{0}, \phi_{0}\right)\left(P_{\alpha_{3}} P_{h \alpha_{4}} \phi_{0}, \phi_{\delta}\right) \\
& =1 \quad \alpha_{1}=\alpha_{2} \text { and } \alpha_{3}=\alpha_{4}=\beta \\
& =0 \text { otherwise. }
\end{aligned}
$$

Therefore

$$
\begin{aligned}
\left(S \phi_{0} \otimes \phi_{0}, \phi_{0} \otimes \phi_{\delta}\right) & =\sum|\sigma(b ; \eta, \beta)|^{2} \quad\left(b \in \mathscr{F}^{\prime}, \eta \in \Gamma\right) \\
& \geqq|\sigma(a ; \alpha, \beta)|^{2} .
\end{aligned}
$$

To show that (3.8) holds, suppose that $\beta=0$ and that $a=(\gamma, g) \notin \Delta \times N\left(G_{0}\right)$. As $A$ satisfies (3.6), $T=\left(A T(d) A^{*}\right)(c)=0$ by Lemma 2.3 and the choice of $g_{0}$, and so

Consequently

$$
\begin{aligned}
\varepsilon^{2} / 4 & \geqq\left\|(T-S) \phi_{0} \otimes \phi_{0}\right\| \\
& \geqq\left|\left(S \phi_{0} \otimes \phi_{0}, \phi_{0} \otimes \phi_{0}\right)\right| \\
& \geqq|\sigma(a ; \alpha, 0)|^{2} .
\end{aligned}
$$

$$
|\theta(a ; \alpha, 0)| \leqq|\theta(a ; \alpha, 0)-\sigma(a ; \alpha, 0)|+|\sigma(a ; \alpha, 0)| \leqq \varepsilon,
$$

and as $\varepsilon>0$ was arbitrary, $\theta(a ; \alpha, 0)=0$.

The last step in the proof consists in showing that (3.7) holds. Suppose that $\beta \neq 0$, and let $T$ be as in the previous paragraph. If $g \notin N\left(G_{0}\right)$, then again $T=0$; and if $g \in N\left(G_{0}\right)$, then $T \in \mathscr{M} \otimes C_{\mathscr{H}}$ (by (3.6) and Lemma 2.3). In any case

$$
\left(T \phi_{0} \otimes \phi_{0}, \phi_{0} \otimes \phi_{\delta}\right)=0
$$

as $\delta \neq 0$, and therefore

$$
\begin{aligned}
\varepsilon^{2} / 4 & \geqq\left\|(S-T) \phi_{0} \otimes \phi_{0}\right\| \\
& \geqq\left|\left((S-T) \phi_{0} \otimes \phi_{0}, \phi_{0} \otimes \phi_{\delta}\right)\right| \\
& \geqq|\sigma(a ; \alpha, \beta)|^{2} .
\end{aligned}
$$

As before, this implies that $\theta(a ; \alpha, \beta)=0$.

4. Examples in type III factors. We continue with the notation of $\$ 3$.

Proposition 4.1. $\mathscr{A}_{p} \otimes \mathscr{B}(G) \cong \mathscr{A}_{p}$ whenever $G$ is the union of an increasing sequence of finite subgroups. 
Proof. Proposition 3.7 and Araki and Woods [2, Definition 3.10 and Corollary 5.5].

LEMMA 4.2. Let $G_{0}$ be a subgroup of $G$ satisfying the conditions in Lemma 3.8 . Then $\mathscr{N} \otimes \mathscr{N}\left(G, G_{0}\right)$ is $M A$ in $\mathscr{A}_{p} \otimes \mathscr{B}(G)$.

Proof. Lemmas 3.2 and 3.8 and [10, p. 455].

Lemma 4.3. Suppose that $G_{0}$ is a subgroup of $G$ which satisfies the condition in Lemma 3.12. Then

$$
\begin{aligned}
& N\left(\mathscr{N} \otimes \mathscr{N}\left(G, G_{0}\right)\right)=\mathscr{A}_{p} \otimes \mathscr{N}\left(G, N\left(G_{0}\right)\right), \\
& N\left(\mathscr{A}_{p} \otimes \mathscr{N}\left(G, G_{0}\right)\right)=\mathscr{A}_{p} \otimes \mathscr{N}\left(G, N\left(G_{0}\right)\right) .
\end{aligned}
$$

Proof. The lemma follows from Definition 3.10, Lemma 3.12, and the fact that $\psi N(\mathscr{P}) \psi^{-1}=N\left(\psi \mathscr{P} \psi^{-1}\right)$ for any subalgebra $\mathscr{P}$ of $\mathscr{A}_{p} \otimes \mathscr{B}(G)$.

Lemma 4.4. For a subgroup $G_{0}$ of $G, \mathscr{A}_{p} \otimes \mathscr{N}\left(G, G_{0}\right)$ is a factor if and only if all nontrivial conjugate classes of $G_{0}$ are infinite.

Proof. The lemma is a consequence of Lemmas 2.1 and 3.9 and [5, pp. 30 and 302].

Proof of Theorem 1. Let $K$ be a countably infinite field which is the union of an increasing sequence of finite subfields (e.g., the algebraic completion of a finite field) and let $K^{*}$ be the multiplicative group of nonzero elements of $K$. Under the operation $(a, b)(c, d)=(a c, a d+b), K^{*} \times K$ becomes a group, say $G$, which is the union of an increasing sequence of finite subgroups. Then all nontrivial conjugate classes of $G$ are infinite and $G_{0}=\{1\} \times K$ is a normal subgroup of $G$ which satisfies the hypothesis of Lemmas 3.8 and 3.12 (cf. [4]). Therefore $\mathscr{N} \otimes \mathscr{N}\left(G, G_{0}\right)$ is a semiregular MA subalgebra of $\mathscr{A}_{p} \otimes \mathscr{B}(G)$. By Proposition 4.1, this completes the proof of Theorem 1.

Proof of Theorem 2. Fix a nonnegative integer $k$. In the proof of [1, Theorem 1], it is shown that there exists an infinite group $G$ which is the union of an increasing sequence of finite subgroups and an abelian subgroup $G_{0}$ of $G$ such that the following hold: (i) $G_{0}$ satisfies the hypothesis of Lemma 3.8, (ii) $N\left(G_{0}\right)$ has a nontrivial conjugate class which is finite, (iii) all nontrivial conjugate classes of $N^{2}\left(G_{0}\right)$ are infinite, and (iv) $G_{0} \subsetneq N\left(G_{0}\right) \varsubsetneqq \cdots \varsubsetneqq N^{2+k}\left(G_{0}\right)=G$, and each of the subgroups $G_{0}, N\left(G_{0}\right), \ldots, N^{1+k}\left(G_{0}\right)$ satisfy the condition of Lemma 3.12. It now follows from Proposition 4.1 and Lemmas 4.2, 4.3, and 4.4 that each $\mathscr{A}_{p}, 0<p<\frac{1}{2}$, contains a 2-semiregular MA subalgebra of improper length $2+k$.

Using the groups and subgroups from the proof of [1, Theorem 2], a similar argument establishes the $n=3$ case of Theorem 2 .

LEMMA 4.5. Let $G_{0}$ be a subgroup of $G$ which satisfies the conditions in Lemma 3.8. Then $\mathscr{N} \otimes \mathscr{M}\left(G, G_{0}\right)$ is $M A$ in $\mathscr{A}_{p} \otimes \mathscr{A}(G)$. 
Proof. By Lemma 3.2 and [10, p. 455], it suffices to show that $\mathscr{M}\left(G, G_{0}\right)$ is MA in $\mathscr{A}(G)$. But that this is so is a consequence of Lemmas 3.8 and 3.9 and [5, p. 57].

REMARKS. (1) The converses of Lemmas 4.2 and 4.5 are also true, but as we will not need them, we omit the proofs.

(2) That $\mathscr{M}\left(G, G_{0}\right)$ is MA in $\mathscr{A}(G)$ under the conditions in Lemma 3.8 is contained in Dixmier [4, Lemme 1].

LemMA 4.6. Suppose that $G_{0}$ is a subgroup of $G$ which satisfies the condition in Lemma 3.12. Then

$$
\begin{aligned}
& N\left(\mathscr{N} \otimes \mathscr{M}\left(G, G_{0}\right)\right)=\mathscr{A}_{p} \otimes \mathscr{M}\left(G, N\left(G_{0}\right)\right), \\
& N\left(\mathscr{A}_{p} \otimes \mathscr{M}\left(G, G_{0}\right)\right)=\mathscr{A}_{p} \otimes \mathscr{M}\left(G, N\left(G_{0}\right)\right) .
\end{aligned}
$$

Proof. First of all, it is clear that $\supset$ holds in both cases. To show that the opposite inclusions also hold, suppose that $U$ is a unitary operator in $\mathscr{A}_{p} \otimes \mathscr{A}(G)$ satisfying one of

$$
\begin{aligned}
& U \mathscr{N} \otimes \mathscr{M}\left(G, G_{0}\right) U^{*}=\mathscr{N} \otimes \mathscr{M}\left(G, G_{0}\right), \\
& U \mathscr{A}_{p} \otimes \mathscr{M}\left(G, G_{0}\right) U^{*}=\mathscr{A}_{p} \otimes \mathscr{M}\left(G, G_{0}\right) .
\end{aligned}
$$

By [5, p. 60], there is a normal *-isomorphism $\Psi$ of $\mathscr{A}_{p} \otimes \mathscr{A}(G)$ into $\mathscr{A}_{p} \otimes \mathscr{B}(G)$ such that $\Psi(A \otimes B)=A \otimes \Phi(B)$ for all $A \in \mathscr{A}_{p}$ and $B \in \mathscr{A}(G)$ ( $\Phi$ as in Lemma 3.9). Then $\Psi(U)$ is a unitary operator in either $N\left(\mathscr{N} \otimes \mathscr{N}\left(G, G_{0}\right)\right)$ or $N\left(\mathscr{A}_{p} \otimes \mathscr{N}\left(G, G_{0}\right)\right)$. Hence $\Psi(U) \in \mathscr{A}_{p} \otimes \mathscr{N}\left(G, N\left(G_{0}\right)\right)$, and so $U \in \mathscr{A}_{p} \otimes \mathscr{M}\left(G, N\left(G_{0}\right)\right)$.

Lemma 4.7. For a subgroup $G_{0}$ of $G, \mathscr{A}_{p} \otimes \mathscr{H}\left(G, G_{0}\right)$ is a factor if and only if all nontrivial conjugate classes of $G_{0}$ are infinite

Proof. Lemma 2.1 and [6, Lemma 5.3.4].

Proof of Theorem 3. Apply Proposition 4.1 and Lemmas 4.5, 4.6, and 4.7 to the groups and subgroups used in [1] (cf. the proof of Theorem 2).

\section{Examples in the type $\mathrm{II}_{1}$ factor.}

LEMMA 5.1. If $G_{0}$ is a subgroup of $G$ satisfying the condition in Lemma 3.12, then $N\left(\mathscr{N}\left(G, G_{0}\right)\right)=\mathscr{N}\left(G, N\left(G_{0}\right)\right)$.

Proof. That $\mathscr{N}\left(G, N\left(G_{0}\right)\right) \subset N\left(\mathcal{N}\left(G, G_{0}\right)\right)$ is trivial. Conversely, suppose that $U$ is a unitary operator in $\mathscr{B}(G)$ such that $U \mathscr{N}\left(G, G_{0}\right) U^{*}=\mathscr{N}\left(G, G_{0}\right)$. Then $I \otimes U$ is a unitary operator in $\mathscr{A}_{p} \otimes \mathscr{B}(G)$ and satisfies

$$
(I \otimes U) \mathscr{A}_{p} \otimes \mathscr{N}\left(G, G_{0}\right)(I \otimes U)^{*}=\mathscr{A}_{p} \otimes \mathscr{N}\left(G, G_{0}\right)
$$

Lemma 4.3 now implies that $I \otimes U \in \mathscr{A}_{p} \otimes \mathscr{N}\left(G, N\left(G_{0}\right)\right)$, and therefore

$$
U \in \mathscr{N}\left(G, N\left(G_{0}\right)\right) .
$$

This proves the lemma. 
Proof of Theorem 4. Apply Lemmas 2.1, 3.8, 3.9 and 5.1 to the groups and subgroups used in [1].

\section{REFERENCES}

1. S. Anastasio, Maximal abelian subalgebras in hyperfinite factors, Amer. J. Math. 87 (1965), 955-971.

2. H. Araki and E. J. Woods, A classification of factors, Publ. Res. Inst. Math. Sci. Ser. A 3 (1968), 51-130.

3. D. Bures, Certain factors constructed as infinite tensor products, Compositio Math. 15 (1963), 169-191.

4. J. Dixmier, Sous-anneaux abélians maximaux dans les facteurs de type fini, Ann. of Math. 59 (1954), 279-286.

5. - Les algèbres d'opérateurs dans l'espace Hilbertien, Gauthier-Villars, Paris, 1957.

6. F. J. Murray and J. von Neumann, On rings of operators. IV, Ann. of Math. 44 (1943), 716-808.

7. R. T. Powers, Representations of uniformly hyperfinite algebras and their associated von Neumann rings, Ann. of Math. 86 (1967), 138-171.

8. L. Pukánszky, Some examples of factors, Publ. Math. Debrecen 4 (1956), 135-156.

9. - On maximal abelian subrings of factors of type II, Canad. J. Math. 12 (1960), 289-296.

10. T. Saitô and J. Tomiyama, Some results on the direct product of $W^{*}$-algebras, Tôhoku Math. J. 12 (1960), 455-458.

11. Sister R. J. Tauer, Maximal abelian subalgebras in finite factors of type II, Trans. Amer. Math. Soc. 114 (1965), 281-308.

12. - Semi-regular maximal abelian subalgebras in hyperfinite factors, Bull. Amer. Math. Soc. 71 (1965), 606-608.

13. - M-semiregular subalgebras in hyperfinite factors, Trans. Amer. Math. Soc. 129 (1967), 530-541.

\section{UNIVERSITY OF BRITISH COLUMBIA,} VANCOUVER, British Columbia, Canada 